\title{
TalkSumm: A Dataset and Scalable Annotation Method for Scientific Paper Summarization Based on Conference Talks
}

\author{
Guy Lev*, Michal Shmueli-Scheuer*, Jonathan Herzig, Achiya Jerbi, David Konopnicki \\ IBM Research, Haifa, Israel \\ \{guylev,shmueli,hjon,davidko\}@il.ibm.com, achiya.jerbi@ibm.com
}

\begin{abstract}
Currently, no large-scale training data is available for the task of scientific paper summarization. In this paper, we propose a novel method that automatically generates summaries for scientific papers, by utilizing videos of talks at scientific conferences. We hypothesize that such talks constitute a coherent and concise description of the papers' content, and can form the basis for good summaries. We collected 1716 papers and their corresponding videos, and created a dataset of paper summaries. A model trained on this dataset achieves similar performance as models trained on a dataset of summaries created manually. In addition, we validated the quality of our summaries by human experts.
\end{abstract}

\section{Introduction}

The rate of publications of scientific papers is increasing and it is almost impossible for researchers to keep up with relevant research. Automatic text summarization could help mitigate this problem. In general, there are two common approaches to summarizing scientific papers: citations-based, based on a set of citation sentences (Nakov et al., 2004; Abu-Jbara and Radev, 2011; Yasunaga et al., 2019), and content-based, based on the paper itself (Collins et al., 2017; Nikola Nikolov and Hahnloser, 2018). Automatic summarization is studied exhaustively for the news domain (Cheng and Lapata, 2016; See et al., 2017), while summarization of scientific papers is less studied, mainly due to the lack of largescale training data. The papers' length and complexity require substantial summarization effort from experts. Several methods were suggested to reduce these efforts (Yasunaga et al., 2019; Collins et al., 2017), still they are not scalable as they require human annotations.

\footnotetext{
* The authors contributed equally.
}

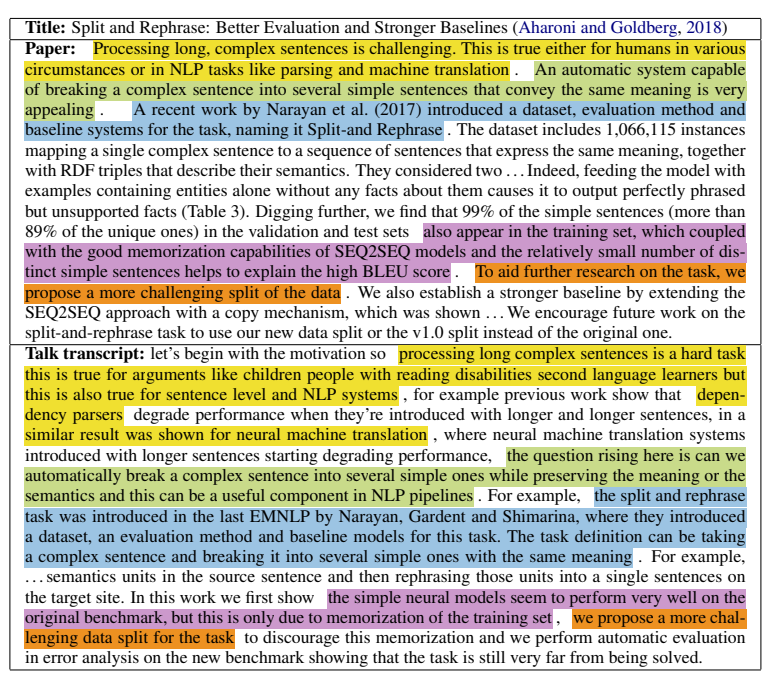

Table 1: Alignment example between a paper's Introduction section and first 2:40 minutes of the talk's transcript. The different colors show corresponding content between the transcript to the written paper.

Recently, academic conferences started publishing videos of talks (e.g., $\mathrm{ACL}^{1}$, EMNLP ${ }^{1}, \mathrm{ICML}^{2}$, and more). In such talks, the presenter (usually a co-author) must describe their paper coherently and concisely (since there is a time limit), providing a good basis for generating summaries. Based on this idea, in this paper, we propose a new method, named TALKSUMM (acronym for Talkbased Summarization), to automatically generate extractive content-based summaries for scientific papers based on video talks. Our approach utilizes the transcripts of video content of conference talks, and treat them as spoken summaries of papers. Then, using unsupervised alignment algorithms, we map the transcripts to the corresponding papers' text, and create extractive summaries. Table 1 gives an example of an alignment between

\footnotetext{
1vimeo.com/aclweb

${ }^{2}$ icml.cc/Conferences/2017/Videos
} 
a paper and its talk transcript (see Table 3 in the appendix for a complete example).

Summaries generated with our approach can then be used to train more complex and datademanding summarization models. Although our summaries may be noisy (as they are created automatically from transcripts), our dataset can easily grow in size as more conference videos are aggregated. Moreover, our approach can generate summaries of various lengths.

Our main contributions are as follows: (1) we propose a new approach to automatically generate summaries for scientific papers based on video talks; (2) we create a new dataset, that contains 1716 summaries for papers from several computer science conferences, that can be used as training data; (3) we show both automatic and human evaluations for our approach. We make our dataset and related code publicly available ${ }^{3}$. To our knowledge, this is the first approach to automatically create extractive summaries for scientific papers by utilizing the videos of conference talks.

\section{Related Work}

Several works focused on generating training data for scientific paper summarization (Yasunaga et al., 2019; Jaidka et al., 2018; Collins et al., 2017; Cohan and Goharian, 2018). Most prominently, the CL-SciSumm shared tasks (Jaidka et al., 2016, 2018) provide a total of 40 human generated summaries; there, a citations-based approach is used, where experts first read citation sentences (citances) that reference the paper being summarized, and then read the whole paper. Then, they create a summary of 150 words on average.

Recently, to mitigate annotation cost, Yasunaga et al. (2019) proposed a method, in which human annotators only read the abstract in addition to citances (not reading the full paper). Using this approach, they generated 1000 summaries, costing $600+$ person-hours. Conversely, we generate summaries, given transcripts of conference talks, in a fully automatic manner, and, thus, our approach is much more scalable. Collins et al. (2017) also aimed at generating labeled data for scientific paper summarization, based on "highlight statements" that authors can provide in some publication venues.

Using external data to create summaries was also proposed in the news domain. Wei and Gao

\footnotetext{
${ }^{3}$ https://github.com/levguy/talksumm
}

(2014, 2015) utilized tweets to decide which sentences to extract from news article.

Finally, alignment between different modalities (e.g., presentation, videos) and text was studied in different domains. Both Kan (2007) and Bahrani and Kan (2013) studied the problem of document to presentation alignment for scholarly documents. Kan (2007) focused on the the discovery and crawling of document-presentation pairs, and a model to align between documents to corresponding presentations. In Bahrani and Kan (2013) they extended previous model to include also visual components of the slides. Aligning video and text was studied mainly in the setting of enriching videos with textual information (Bojanowski et al., 2015; Malmaud et al., 2015; Zhu et al., 2015). Malmaud et al. (2015) used HMM to align ASR transcripts of cooking videos and recipes text for enriching videos with instructions. Zhu et al. (2015) utilized books to enrich videos with descriptive explanations. Bojanowski et al. (2015) proposed to align video and text by providing a time stamp for every sentence. The main difference between these works and ours is in the alignment being used to generate textual training data in our case, rather than to enrich videos.

\section{The TALKSumm Dataset}

\subsection{Data Collection}

Recently, many computer science academic associations including ACL, ACM, IMLS and more, have started recording talks in different conferences, e.g., ACL, NAACL, EMNLP, and other colocated workshops. A similar trend occurs in other domains such as Physics ${ }^{4}$, Biology ${ }^{5}$, etc.

In a conference, each speaker (usually a coauthor) presents their paper given a timeframe of 15-20 minutes. Thus, the talk must be coherent and concentrate on the most important aspects of a paper. Hence, the talk can be considered as a summary of the paper, as viewed by its authors, and is much more comprehensive than the abstract, which is written by the authors as well.

In this work, we focused on NLP and ML conferences, and analyzed 1716 video talks from ACL, NAACL, EMNLP, SIGDIAL (2015-2018), and ICML (2017-2018). We downloaded the videos and extracted the speech data. Then, via

\footnotetext{
${ }^{4}$ www. cleoconference.org

5 igem.org/Videos/Lecture_Videos
} 
a publicly available ASR service ${ }^{6}$, we extracted transcripts of the speech, and based on the video metadata (e.g., title), we retrieved the corresponding paper (in PDF format). We used ScienceParse $^{7}$ to extract the text of the paper, and applied a simple processing in order to filter-out some noise (e.g. lines starting with the word "Copyright"). At the end of this process, the text of each paper is associated with the transcript of the corresponding talk.

\subsection{Dataset Generation}

The transcript itself cannot serve as a good summary for the corresponding paper, as it constitutes only one modality of the talk (which also consists of slides, for example), and hence cannot stand by itself and form a coherent written text. Thus, to create an extractive paper summary based on the transcript, we model the alignment between spoken words and sentences in the paper, assuming the following generative process: During the talk, the speaker generates words for describing verbally sentences from the paper, one word at each time step. Thus, at each time step, the speaker has a single sentence from the paper in mind, and produces a word that constitutes a part of its verbal description. Then, at the next time-step, the speaker either stays with the same sentence, or moves on to describing another sentence, and so on. Thus, given the transcript, we aim to retrieve those "source" sentences and use them as the summary. The number of words uttered to describe each sentence can serve as importance score, indicating the amount of time the speaker spent describing the sentence. This enables to control the summary length by considering only the most important sentences up to some threshold.

We use an HMM to model the assumed generative process. The sequence of spoken words is the output sequence. Each hidden state of the HMM corresponds to a single paper sentence. We heuristically define the HMM's probabilities as follows.

Denote by $Y(1: T)$ the spoken words, and by $S(t) \in\{1, \ldots, K\}$ the paper sentence index at time-step $t \in\{1, \ldots, T\}$. Similarly to Malmaud et al. (2015), we define the emission probabilities

\footnotetext{
${ }^{6}$ www. ibm. com/watson/services/ speech-to-text/

${ }^{7}$ github.com/allenai/science-parse
}

to be:

$$
p(Y(t)=y \mid S(t)=k) \propto \max _{w \in \operatorname{words}(k)} \operatorname{sim}(y, w)
$$

where $\operatorname{words}(k)$ is the set of words in the $k^{\prime}$ th sentence, and sim is a semantic-similarity measure between words, based on word-vector distance. We use pre-trained GloVe (Pennington et al., 2014) as the semantic vector representations for words.

As for the transition probabilities, we must model the speaker's behavior and the transitions between any two sentences in the paper. This is unlike the simpler setting in Malmaud et al. (2015), where transition is allowed between consecutive sentences only. To do so, denote the entries of the transition matrix by $T(k, l)=p(S(t+$ $1)=l \mid S(t)=k)$. We rely on the following assumptions: (1) $T(k, k)$ (the probability of staying in the same sentence at the next time-step) is relatively high. (2) There is an inverse relation between $T(k, l)$ and $|l-k|$, i.e., it is more probable to move to a nearby sentence than jumping to a farther sentence. (3) $S(t+1)>S(t)$ is more probable than the opposite (i.e., transition to a later sentence is more probable than to an earlier one). Although these assumptions do not perfectly reflect reality, they are a reasonable approximation in practice.

Following these assumptions, we define the HMM's transition probability matrix. First, define the stay-probability as $\alpha=\max (\delta(1-$ $\left.\left.\frac{K}{T}\right), \epsilon\right)$, where $\delta, \epsilon \in(0,1)$. This choice of stayprobability is inspired by Malmaud et al. (2015), using $\delta$ to fit it to our case where transitions between any two sentences are allowed, and $\epsilon$ to handle rare cases where $K$ is close to, or even larger than $T$. Then, for each sentence index $k \in\{1, \ldots, K\}$, we define:

$$
\begin{gathered}
T(k, k)=\alpha \\
T(k, k+j)=\beta_{k} \cdot \lambda^{j-1}, \quad j \geq 1 \\
T(k, k-j)=\gamma \cdot \beta_{k} \cdot \lambda^{j-1}, \quad j \geq 1
\end{gathered}
$$

where $\lambda, \gamma, \beta_{k} \in(0,1), \lambda$ and $\gamma$ are factors reflecting assumptions (2) and (3) respectively, and for all $k, \beta_{k}$ is normalized s.t. $\sum_{l=1}^{K} T(k, l)=1$. The values of $\lambda, \gamma, \delta$ and $\epsilon$ were fixed throughout our experiments at $\lambda=0.75, \gamma=0.5$, $\delta=0.33$ and $\epsilon=0.1$. The average value of $\alpha$, across all papers, was around 0.3. The values of 
these parameters were determined based on evaluation over manually-labeled alignments between the transcripts and the sentences of a small set of papers.

Finally, we define the start-probabilities assuming that the first spoken word must be conditioned on a sentence from the Introduction section, hence $p(S(1))$ is defined as a uniform distribution over the Introduction section's sentences.

Note that sentences which appear in the Abstract, Related Work, and Acknowledgments sections of each paper are excluded from the HMM's hidden states, as we observed that presenters seldom refer to them.

To estimate the MAP sequence of sentences, we apply the Viterbi algorithm. The sentences in the obtained sequence are the candidates for the paper's summary. For each sentence $s$ appearing in this sequence, denote by count $(s)$ the number of time-steps in which this sentence appears. Thus, count $(s)$ models the number of words generated by the speaker conditioned on $s$, and, hence, can be used as an importance score. Given a desired summary length, one can draw a subset of topranked sentences up to this length.

\section{Experiments}

\subsection{Experimental Setup}

Data For Evaluation We evaluate the quality of our dataset generation method by training an extractive summarization model, and evaluating this model on a human-generated dataset of scientific paper summaries. For this, we choose the CL-SciSumm shared task (Jaidka et al., 2016, 2018), as this is the most established benchmark for scientific paper summarization. In this dataset, experts wrote summaries of 150 words length on average, after reading the whole paper. The evaluation is on the same test data used by Yasunaga et al. (2019), namely 10 examples from CL-SciSumm 2016, and 20 examples from CLSciSumm 2018 as validation data.

Training Data Using the HMM importance scores, we create four training sets, two with fixed-length summaries (150 and 250 words), and two with fixed ratio between summary and paper lengths (0.3 and 0.4). We train models on each training set, and select the model yielding the best performance on the validation set (evaluation is always done with generating a 150 -words sum-

\begin{tabular}{l||l|c|c|c}
\hline \hline Model & $2-\mathrm{R}$ & $2-\mathrm{F}$ & $3-\mathrm{F}$ & SU4-F \\
TALKSUMM-HyBRID & $\mathbf{3 5 . 0 5}$ & $\mathbf{3 4 . 1 1}$ & $\mathbf{2 7 . 1 9}$ & $\mathbf{2 4 . 1 3}$ \\
TALKSUMM-ONLY & 22.77 & 21.94 & 15.94 & 12.55 \\
\hline GCN HYBRID 2* & 32.44 & 30.08 & 23.43 & 23.77 \\
GCN CITED TEXT SPANS* & 25.16 & 24.26 & 18.79 & 17.67 \\
ABSTRACT* & 29.52 & 29.4 & 23.16 & 23.34 \\
\hline \hline
\end{tabular}

Table 2: ROUGE scores on the CL-SciSumm 2016 test benchmark. *: results from Yasunaga et al. (2019).

mary).

Summarization Model We train an extractive summarization model on our TALKSUMM dataset, using the extractive variant of Chen and Bansal (2018). We test two summary generation approaches, similarly to Yasunaga et al. (2019). First, for TALKSUMM-ONLY, we generate a 150words summary out of the top-ranked sentences extracted by our trained model (sentences from the Acknowledgments section are omitted, in case the model extracts any). In the second approach, a 150 -words summary is created by augmenting the abstract with non-redundant sentences extracted by our model, similarly to the "Hybrid 2" approach of Yasunaga et al. (2019). We perform early-stopping and hyper-parameters tuning using the validation set.

Baselines We compare our results to SCISUMMNET (Yasunaga et al., 2019) trained on 1000 scientific papers summarized by human annotators. As we use the same test set as in Yasunaga et al. (2019), we directly compare their reported model performance to ours, including their ABSTRACT baseline which takes the abstract to be the paper's summary.

\subsection{Results}

Automatic Evaluation Table 2 summarizes the results: both GCN CITED TEXT SPANS and TALKSUMM-ONLY models, are not able to obtain better performance than ABSTRACT ${ }^{8}$. However, for the Hybrid approach, where the abstract is augmented with sentences from the summaries emitted by the models, our TALKSUMM-HYBRID outperforms both GCN HYBRID 2 and ABSTRACT. Importantly, our model, trained on automaticallygenerated summaries, performs on par with models trained over SCISUMMNET, in which training data was created manually.

\footnotetext{
${ }^{8}$ While the abstract was input to GCN CITED TEXT SPANS, it was excluded from TALKSUMM-ONLY.
} 
Human Evaluation We conduct a human evaluation of our approach with support from authors who presented their papers in conferences. As our goal is to test more comprehensive summaries, we generated summaries composed of 30 sentences (approximately $15 \%$ of a long paper). We randomly selected 15 presenters from our corpus and asked them to perform two tasks, given the generated summary of their paper: (1) for each sentence in the summary, we asked them to indicate whether they considered it when preparing the talk (yes/no question); (2) we asked them to globally evaluate the quality of the summary (1-5 scale, ranging from very bad to excellent, 3 means good). For the sentence-level task (1), $73 \%$ of the sentences were considered while preparing the talk. As for the global task (2), the quality of the summaries was 3.73 on average, with standard deviation of 0.725 . These results validate the quality of our generation method.

\section{Conclusion}

We propose a novel automatic method to generate training data for scientific papers summarization, based on conference talks given by authors. We show that the a model trained on our dataset achieves competitive results compared to models trained on human generated summaries, and that the dataset quality satisfies human experts. In the future, we plan to study the effect of other video modalities on the alignment algorithm. We hope our method and dataset will unlock new opportunities for scientific paper summarization.

\section{References}

Amjad Abu-Jbara and Dragomir Radev. 2011. Coherent citation-based summarization of scientific papers. In Proceedings of the 49th Annual Meeting of the Association for Computational Linguistics: $\mathrm{Hu}$ man Language Technologies - Volume 1, HLT '11, pages 500-509.

Roee Aharoni and Yoav Goldberg. 2018. Split and rephrase: Better evaluation and stronger baselines. In Proceedings of the 56th Annual Meeting of the Association for Computational Linguistics (Volume 2: Short Papers), pages 719-724. Association for Computational Linguistics.

Bamdad Bahrani and Min-Yen Kan. 2013. Multimodal alignment of scholarly documents and their presentations. In Proceedings of the 13th ACM/IEEE-CS Joint Conference on Digital Libraries, JCDL '13, pages 281-284.
Piotr Bojanowski, Remi Lajugie, Edouard Grave, Francis Bach, Ivan Laptev, Jean Ponce, and Cordelia Schmid. 2015. Weakly-supervised alignment of video with text. In The IEEE International Conference on Computer Vision (ICCV).

Yen-Chun Chen and Mohit Bansal. 2018. Fast abstractive summarization with reinforce-selected sentence rewriting. In Proceedings of the 56th Annual Meeting of the Association for Computational Linguistics (Volume 1: Long Papers), pages 675-686. Association for Computational Linguistics.

Jianpeng Cheng and Mirella Lapata. 2016. Neural summarization by extracting sentences and words. In Proceedings of the 54th Annual Meeting of the Association for Computational Linguistics (Volume 1: Long Papers), pages 484-494.

Arman Cohan and Nazli Goharian. 2018. Scientific document summarization via citation contextualization and scientific discourse. International Journal on Digital Libraries, pages 287-303.

Ed Collins, Isabelle Augenstein, and Sebastian Riedel. 2017. A supervised approach to extractive summarisation of scientific papers. In Proceedings of the 21 st Conference on Computational Natural Language Learning (CoNLL 2017), pages 195-205.

Kokil Jaidka, Muthu Kumar Chandrasekaran, Sajal Rustagi, and Min-Yen Kan. 2016. Overview of the cl-scisumm 2016 shared task. In In Proceedings of Joint Workshop on Bibliometric-enhanced Information Retrieval and NLP for Digital Libraries (BIRNDL 2016).

Kokil Jaidka, Michihiro Yasunaga, Muthu Kumar Chandrasekaran, Dragomir Radev, and Min-Yen Kan. 2018. The cl-scisumm shared task 2018: Results and key insights. In Proceedings of the $3 \mathrm{rd}$ Joint Workshop on Bibliometric-enhanced Information Retrieval and Natural Language Processing for Digital Libraries (BIRNDL).

Min-Yen Kan. 2007. Slideseer: A digital library of aligned document and presentation pairs. In Proceedings of the 7th ACM/IEEE-CS Joint Conference on Digital Libraries, JCDL '07, pages 81-90.

Jonathan Malmaud, Jonathan Huang, Vivek Rathod, Nicholas Johnston, Andrew Rabinovich, and Kevin Murphy. 2015. What's cookin'? interpreting cooking videos using text, speech and vision. In Proceedings of the 2015 Conference of the North American Chapter of the Association for Computational Linguistics: Human Language Technologies, pages 143-152. Association for Computational Linguistics.

Preslav I. Nakov, Ariel S. Schwartz, and Marti A. Hearst. 2004. Citances: Citation sentences for semantic analysis of bioscience text. In In Proceedings of the SIGIR?04 workshop on Search and Discovery in Bioinformatics. 
Michael Pfeiffer Nikola Nikolov and Richard Hahnloser. 2018. Data-driven summarization of scientific articles. In Proceedings of the Eleventh International Conference on Language Resources and Evaluation (LREC 2018).

Jeffrey Pennington, Richard Socher, and Christopher D. Manning. 2014. Glove: Global vectors for word representation. In In EMNLP.

Abigail See, Peter J. Liu, and Christopher D. Manning. 2017. Get to the point: Summarization with pointergenerator networks. In Proceedings of the 55th Annual Meeting of the Association for Computational Linguistics (Volume 1: Long Papers), pages 10731083.

Zhongyu Wei and Wei Gao. 2014. Utilizing microblogs for automatic news highlights extraction. In Proceedings of COLING 2014, the 25th International Conference on Computational Linguistics: Technical Papers, pages 872-883. Dublin City University and Association for Computational Linguistics.

Zhongyu Wei and Wei Gao. 2015. Gibberish, assistant, or master?: Using tweets linking to news for extractive single-document summarization. In Proceedings of the 38th International ACM SIGIR Conference on Research and Development in Information Retrieval, SIGIR '15, pages 1003-1006.

Michihiro Yasunaga, Jungo Kasai, Rui Zhang, Alexander Fabbri, Irene Li, Dan Friedman, and Dragomir Radev. 2019. Scisummnet: A large annotated corpus and content-impact models for scientific paper summarization with citation networks. In Proceedings of $A A A I 2019$.

Yukun Zhu, Ryan Kiros, Rich Zemel, Ruslan Salakhutdinov, Raquel Urtasun, Antonio Torralba, and Sanja Fidler. 2015. Aligning books and movies: Towards story-like visual explanations by watching movies and reading books. In The IEEE International Conference on Computer Vision (ICCV).

\section{A A Detailed Example}

This section elaborates on the example presented in Table 1. Table 3 extends Table 1 by showing the manually-labeled alignment between the complete text of the paper's Introduction section, and the corresponding transcript. Table 4 shows the alignment obtained using the HMM. Each row in this table corresponds to an interval of consecutive time-steps (i.e., a sub-sequence of the transcript) in which the same paper sentence was selected by the Viterbi algorithm. The first column (Paper Sentence) shows the selected sentences; The second column (ASR transcript) shows the transcript obtained by the ASR system; The third column (Human transcript) shows the manually corrected transcript, which is provided for readability

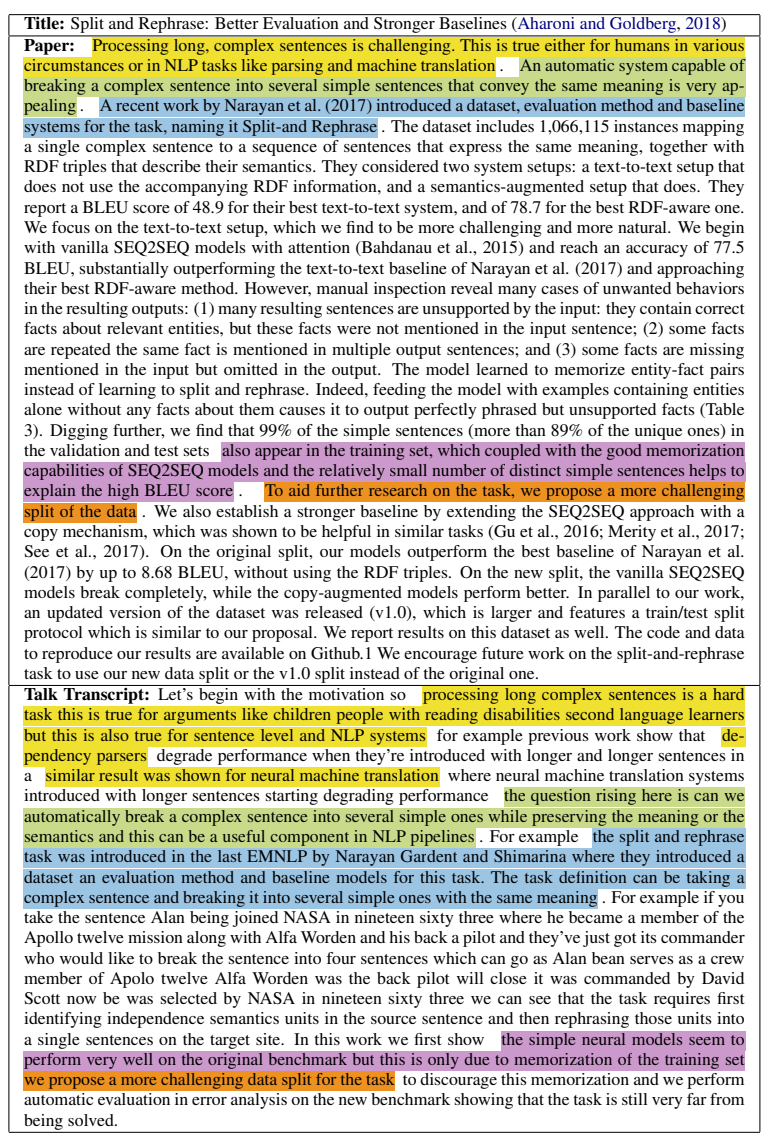

Table 3: Alignment example between a paper's Introduction section and first 2:40 minutes of the talk's transcript. The different colors show corresponding content between the transcript to the written paper. This is the full-text version of the example shown in Table 1.

(our model predicted the alignment based on the raw ASR output); Finally, the forth column shows whether our model has correctly aligned a paper sentence with a sub-sequence of the transcript. Rows with no values in this column correspond to transcript sub-sequences which were not associated with any paper sentence in the manuallylabeled alignment. 


\begin{tabular}{|c|c|c|c|}
\hline Paper Sentence & ASR transcript & Human transcript & \\
\hline $\begin{array}{l}\text { Processing long, complex } \\
\text { sentences is challenging. }\end{array}$ & $\begin{array}{l}\text { base begin motivation } \\
\text { processing long complex } \\
\text { sentences hard task }\end{array}$ & $\begin{array}{l}\text { Let's begin with the moti- } \\
\text { vation so processing long } \\
\text { complex sentences is a } \\
\text { hard task }\end{array}$ & $\checkmark$ \\
\hline $\begin{array}{l}\text { This is true either for hu- } \\
\text { mans in various circum- } \\
\text { stances or in NLP tasks } \\
\text { like parsing and machine } \\
\text { translation. }\end{array}$ & $\begin{array}{l}\text { true arguments like chil- } \\
\text { dren people reading dis- } \\
\text { abilities second language } \\
\text { learners also true first } \\
\text { sentence level p system }\end{array}$ & $\begin{array}{l}\text { this is true for arguments } \\
\text { like children people with } \\
\text { reading disabilities sec- } \\
\text { ond language learners but } \\
\text { this is also true for sen- } \\
\text { tence level and NLP sys- } \\
\text { tems }\end{array}$ & $\checkmark$ \\
\hline $\begin{array}{l}\text { A recent work by } \\
\text { Narayan et al. (2017) } \\
\text { introduced a dataset, } \\
\text { evaluation method and } \\
\text { baseline systems for the } \\
\text { task, naming it Split-and } \\
\text { Rephrase. }\end{array}$ & $\begin{array}{l}\text { previous work show data } \\
\text { tendency parsers great } \\
\text { performance introduced } \\
\text { longer longer sentences }\end{array}$ & $\begin{array}{l}\text { previous work show that } \\
\text { dependency parsers de- } \\
\text { grade performance when } \\
\text { they're introduced with } \\
\text { longer and longer sen- } \\
\text { tences }\end{array}$ & $x$ \\
\hline $\begin{array}{l}\text { This is true either for hu- } \\
\text { mans in various circum- } \\
\text { stances or in NLP tasks } \\
\text { like parsing and machine } \\
\text { translation. }\end{array}$ & $\begin{array}{l}\text { similar results showing } \\
\text { new machine translation } \\
\text { new machine translation }\end{array}$ & $\begin{array}{l}\text { similar result was shown } \\
\text { for neural machine trans- } \\
\text { lation where neural ma- } \\
\text { chine translation }\end{array}$ & $\checkmark$ \\
\hline $\begin{array}{l}\text { An automatic system ca- } \\
\text { pable of breaking a com- } \\
\text { plex sentence into sev- } \\
\text { eral simple sentences that } \\
\text { convey the same meaning } \\
\text { is very appealing. }\end{array}$ & $\begin{array}{l}\text { systems introduced } \\
\text { longer sentences starting } \\
\text { performance question ris- } \\
\text { ing automatically break } \\
\text { complex sentence several } \\
\text { simple ones preserving } \\
\text { meaning semantics useful } \\
\text { company p like example }\end{array}$ & $\begin{array}{l}\text { systems introduced with } \\
\text { longer sentences starting } \\
\text { degrading performance } \\
\text { the question rising here } \\
\text { is can we automatically } \\
\text { break a complex sentence } \\
\text { into several simple ones } \\
\text { while preserving the } \\
\text { meaning or the semantics } \\
\text { and this can be a use- } \\
\text { ful component in NLP } \\
\text { pipelines for example }\end{array}$ & $\checkmark$ \\
\hline $\begin{array}{l}\text { A recent work by } \\
\text { Narayan et al. (2017) } \\
\text { introduced a dataset, } \\
\text { evaluation method and } \\
\text { baseline systems for the } \\
\text { task, naming it Split-and } \\
\text { Rephrase. }\end{array}$ & $\begin{array}{l}\text { leader task introduced } \\
\text { last 'll bynari guard going } \\
\text { marina introduced data } \\
\text { sets evaluation method } \\
\text { baseline models task }\end{array}$ & $\begin{array}{l}\text { the split and rephrase task } \\
\text { was introduced in the } \\
\text { last EMNLP by Narayan } \\
\text { Gardent and Shimarina } \\
\text { where they introduced } \\
\text { a dataset an evaluation } \\
\text { method and baseline } \\
\text { models for this task }\end{array}$ & $\checkmark$ \\
\hline $\begin{array}{l}\text { An automatic system ca- } \\
\text { pable of breaking a com- } \\
\text { plex sentence into sev- } \\
\text { eral simple sentences that } \\
\text { convey the same meaning } \\
\text { is very appealing. }\end{array}$ & $\begin{array}{l}\text { phoenician taking com- } \\
\text { plex sentences break } \\
\text { several simple ones } \\
\text { example take sentence } \\
\text { alan joined nasa nine- } \\
\text { teen sixty three became } \\
\text { member apollo twelve } \\
\text { mission along word } \\
\text { inspect pilot got com- } \\
\text { mander would like break } \\
\text { sentence sentences go } \\
\text { alan serves crew member } \\
\text { twelve word better polls } \\
\text { commanded david scott } \\
\text { selected nasa nineteen } \\
\text { sixty three }\end{array}$ & $\begin{array}{l}\text { the task definition can } \\
\text { be taking a complex } \\
\text { sentence and break it } \\
\text { into several simple ones } \\
\text { for example if you take } \\
\text { the sentence Alan being } \\
\text { joined NASA in nineteen } \\
\text { sixty three where he } \\
\text { became a member of the } \\
\text { Apollo twelve mission } \\
\text { along with Alfa Worden } \\
\text { and his back a pilot and } \\
\text { they've just got its com- } \\
\text { mander who would like } \\
\text { to break the sentence into } \\
\text { four sentences which can } \\
\text { go as Alan bean serves as } \\
\text { a crew member of Apolo } \\
\text { twelve Alfa Worden was } \\
\text { the back pilot will close } \\
\text { it was commanded by } \\
\text { David Scott now be was } \\
\text { selected by NASA in } \\
\text { nineteen sixty three }\end{array}$ & \\
\hline $\begin{array}{l}\text { A recent work by } \\
\text { Narayan et al. (2017) } \\
\text { introduced a dataset, } \\
\text { evaluation method and } \\
\text { baseline systems for the } \\
\text { task, naming it Split-and } \\
\text { Rephrase. }\end{array}$ & $\begin{array}{l}\text { see task requires first } \\
\text { identifying independence } \\
\text { imagic units }\end{array}$ & $\begin{array}{l}\text { we can see that the task } \\
\text { requires first identifying } \\
\text { independence semantics } \\
\text { units }\end{array}$ & \\
\hline $\begin{array}{l}\text { The dataset includes } \\
1,066,115 \text { instances map- } \\
\text { ping a single complex } \\
\text { sentence to a sequence of } \\
\text { sentences that express the } \\
\text { same meaning, together } \\
\text { with RDF triples that } \\
\text { describe their semantics. }\end{array}$ & $\begin{array}{l}\text { source sentence rephras- } \\
\text { ing units single sentences } \\
\text { target }\end{array}$ & $\begin{array}{l}\text { in the source sentence } \\
\text { and then rephrasing those } \\
\text { units into a single sen- } \\
\text { tences on the target site }\end{array}$ & \\
\hline $\begin{array}{l}\text { Digging further, we find } \\
\text { that } 99 \% \text { of the sim- } \\
\text { ple sentences (more than } \\
89 \% \text { of the unique ones) } \\
\text { in the validation and test } \\
\text { sets also appear in the } \\
\text { training set, which cou- } \\
\text { pled with the good mem- } \\
\text { orization capabilities of } \\
\text { SEQ2SEQ models and } \\
\text { the relatively small num- } \\
\text { ber of distinct simple sen- } \\
\text { tences helps to explain } \\
\text { the high BLEU score. }\end{array}$ & $\begin{array}{l}\text { work first show simple } \\
\text { neural models seem per- } \\
\text { form well original bench- } \\
\text { mark due memorization } \\
\text { training set }\end{array}$ & $\begin{array}{l}\text { In this work we first } \\
\text { show the simple neural } \\
\text { models seem to perform } \\
\text { very well on the origi- } \\
\text { nal benchmark but this is } \\
\text { only due to memorization } \\
\text { of the training set }\end{array}$ & $\checkmark$ \\
\hline $\begin{array}{l}\text { To aid further research on } \\
\text { the task, we propose a } \\
\text { more challenging split of } \\
\text { the data. }\end{array}$ & 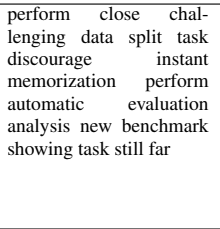 & $\begin{array}{l}\text { we propose a more chal- } \\
\text { lenging data split for the } \\
\text { task to discourage this } \\
\text { memorization and we } \\
\text { perform automatic eval- } \\
\text { uation in error analysis } \\
\text { on the new benchmark } \\
\text { showing that the task is } \\
\text { still very far from being } \\
\text { solved }\end{array}$ & $\checkmark$ \\
\hline
\end{tabular}

Table 4: Alignment obtained using the HMM, for the Introduction section and first 2:40 minutes of the2131 video's transcript. 